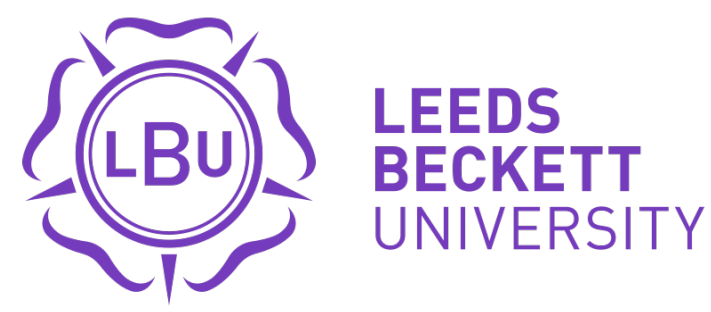

Citation:

Ghaffari, A and Zarachoff, M and Sheikh Akbari, A and Shaghouei, E (2019) Intensity Separation based Iris Recognition Method using Curvelets and PCA. 2019 8th Mediterranean Conference on Embedded Computing (MECO). ISSN 2637-9511 DOI: https://doi.org/10.1109/MECO.2019.8760170

Link to Leeds Beckett Repository record:

https://eprints.leedsbeckett.ac.uk/id/eprint/5902/

Document Version:

Article (Accepted Version)

(C) 2019 IEEE. Personal use of this material is permitted. Permission from IEEE must be obtained for all other uses, in any current or future media, including reprinting/republishing this material for advertising or promotional purposes, creating new collective works, for resale or redistribution to servers or lists, or reuse of any copyrighted component of this work in other works.

The aim of the Leeds Beckett Repository is to provide open access to our research, as required by funder policies and permitted by publishers and copyright law.

The Leeds Beckett repository holds a wide range of publications, each of which has been checked for copyright and the relevant embargo period has been applied by the Research Services team.

We operate on a standard take-down policy. If you are the author or publisher of an output and you would like it removed from the repository, please contact us and we will investigate on a case-by-case basis.

Each thesis in the repository has been cleared where necessary by the author for third party copyright. If you would like a thesis to be removed from the repository or believe there is an issue with copyright, please contact us on openaccess@leedsbeckett.ac.uk and we will investigate on a case-by-case basis. 


\title{
Intensity Separation based Iris Recognition Method using Curvelets and PCA
}

\author{
Ahmad Ghaffari, Matthew Zarachoff, Akbar Sheikh-Akbari, and Ebrahim Shaghouei \\ Leeds Beckett University, School of Computing, Creative Technologies \& Engineering \\ Caedmon Hall, Leeds, LS6 3QS, United Kingdom \\ Email: A.Ghaffari6582@student.leedsbeckett.ac.uk
}

\begin{abstract}
This paper presents a PCA-based iris recognition method called Intensity Separation Curvelet based PCA (ISCPCA). The proposed method uses Canny Edge detection and the Hough transform to extract and rectangularize the iris from the input eye image. The second generation Fast Digital Curvelet Transform (FDCT) is then applied to the resulting image, dividing it into its subbands. The resulting complex subbands coefficients within the same level are concatenated, generating two single frames. The coefficients in each resulting frame are then normalized and evenly divided into a preselected number of bands. The coefficient matrices within each frame are then vectorized and concatenated, generating a single $2 \mathrm{D}$ matrix. Conventional PCA is then performed on the resulting 2D matrix extracting its eigenvectors which are used for iris matching. The Euclidean distance is used as a measure to quantify the closeness of different iris images. Experimental results on images from the CASIA-Iris-Interval benchmark eye image dataset show that the proposed ISC-PCA technique significantly outperforms the state of the art PCA based methods, and achieves competitive results to those of the learning based techniques.
\end{abstract}

\section{INTRODUCTION}

The application of iris images for human identification has shown significant potential, as the iris patterns of individuals are unique, even between the two eyes of a single person [1]. Iris recognition is performed by capturing the image of an eye, extracting the iris from the image, and calculating descriptors from the resulting iris that can be used for matching [2]. Many iris recognition techniques have been reported by researchers over the last three decades [3]. These methods can generally be classified into one of two main categories: statistical based and learning based methods. Statistical methods extract some features from the input iris image and compare these features directly by some sort of distance measures to assess and find its closest match. Example of these techniques include Principal Component Analysis (PCA) [3], Wavelet [4], Curvelet [5], Gabor filter [6], and non-orthogonal transform [7] based techniques. Learning based methods use one more statistical based feature extraction algorithms. This is followed by a classifier such as a neural network [8] or support vector machine [9] to perform the matching. PCA has been widely used for feature extraction and dimensionality reduction in conjunction with both statistical and learning based algorithms.

Although various learning based iris recognition techniques have been reported in the literature that provide high accuracy, they require significantly high computational power, which may not be available on low power mobile devices.
In addition, with advances in technology and the security based need for human identification on the go, demand for low power, portable (e.g. embedded mobile systems) iris recognition systems has increased. The success of PCA for hyperspectral image classification [10] has inspired the authors to propose the Intensity Separation Curvelet based PCA (ISCPCA) iris recognition technique, which requires significantly less computational power that learning based techniques, while simultaneously providing competitive matching accuracy. Unlike other Curvelet and PCA based iris recognition techniques, ISC-PCA is unique in that it divides the Curvelet subbands' coefficients into several matrices, resembling a multispectral image and enriching the data available for feature extraction. The proposed method first segments the input eye image, extracting its iris. The resulting iris is then rectangularized and histogram equalized. The second generation Fast Digital Curvelet Transform (FDCT) [11] is then applied to the resulting image, splitting the image into its Curvelet subbands. The real and imaginary components for each resulting Curvelet level subbands are individually concatenated and normalized, generating two matrices per level. Each resulting matrix is then evenly split into a set of bands, generating a three-dimensional matrix called a frame. The frame is then converted to a twodimensional matrix, where each column represents a band within the frame. Principal Component Analysis (PCA) is then applied to the resulting matrix, extracting its eigenvectors as features. The Euclidean distance is used as a criterion to evaluate the distance between the eigenvectors of two iris images. The images from the CASIA-Iris-Interval dataset have been used to assess and compare the performance of the proposed ISC-PCA algorithm to both statistical and learning based techniques. Experimental results show that the proposed ISC-PCA method substantially outperforms single image PCA and the eigenfaces [12] techniques. These results also show that the proposed algorithm gives highly competitive performance to those of learning based techniques, including PCA with Neural Network [13] and Modified Convolutional Neural Network [14] at significantly lower computational cost. The rest of the paper is organized as follows: Section II introduces the proposed ISC-PCA algorithm, Section III discusses the benchmark CASIA-Iris-Interval eye image dataset and the experimental results, and Section IV concludes the paper. 

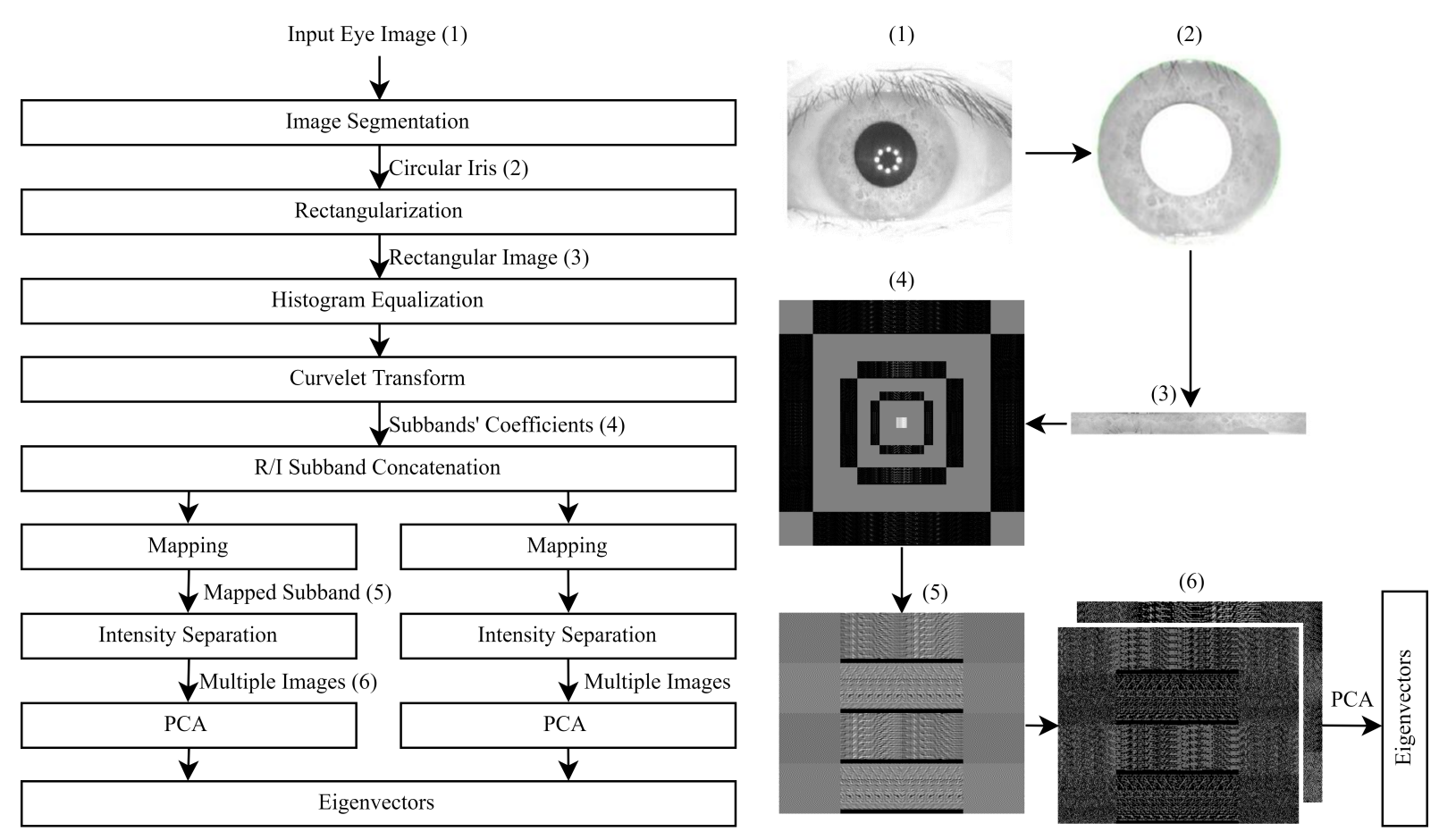

Fig. 1. Block diagram for the proposed Intensity Separation Curvelet based PCA (ISC-PCA) technique.

\section{Proposed Intensity Separation Curvelet based PCA METHOD}

Fig. 1 shows a block diagram of the proposed Intensity Separation Curvelet based PCA (ISC-PCA) method. The proposed method first extracts the iris from the input 8-bit grayscale eye image using Canny Edge detection followed by the Hough transform as specified in [15]. The resulting rectangular iris image is then histogram equalized to increase its contrast. This is accomplished by first calculating its Probability Mass Function (PMF), as seen in (1):

$$
P M F_{X}\left(x_{k}\right)=P\left(X=x_{k}\right) \text { for } k=0,1, \ldots, 255
$$

where $X=x_{0}, x_{1}, \ldots, x_{255}$ represent the iris image's pixel values and $P M F_{X}\left(x_{k}\right)$ is the probability of pixels in bin $\mathrm{k}$. The resulting PMF is then used to calculate the Cumulative Distribution Function (CDF) as seen in (2):

$$
C D F_{X}(k)=P\left(X \leq x_{k}\right) \text { for } k=0,1, \ldots, 255
$$

where $C D F_{X}(k)$ is the cumulative probability of $X \leq x_{k}$. Finally, all input iris image pixel values are mapped to new values using the resulting CDF. The proposed method then applies the second generation Fast Digital Curvelet Transform (FDCT) using the wrapping variant [11] on the resulting histogram equalized iris image, splitting the image into its subbands. The resulting subbands' coefficients are complex numbers. Within each Curvelet decomposition level, the real and imaginary parts of the resulting Curvelet subbands' coefficients are separately concatenated, generating two coefficient matrices per level. The resulting matrices are named $C_{m R / I}$, where $C$ is the matrix, $m$ represents the decomposition level, and $R$ or $I$ indicate the real or imaginary components, respectively. The original coefficients $p \in C_{m R / I}$ are then mapped to new values $p^{\prime}$ in $[0,1]$ domain using (3):

$$
p^{\prime}=\frac{p-\min \left(C_{m R / I}\right)}{\max \left(C_{m R / I}\right)-\min \left(C_{m R / I}\right)}
$$

The proposed method then applies an even partitioning algorithm to split each resulting matrix into a set of multiple images called a frame. Each frame is comprised of $N$ matrices, which are generated from $N-1$ boundary values called $B=\left[b_{1}, b_{2}, \ldots, b_{N-1}\right]$. In the even partitioning algorithm, the boundary values are calculated using (4):

$$
b_{n}=n / N \text { for } n=1,2, \ldots,(N-1)
$$

A frame $F$ consisting of $N$ zero matrices of the size of $C_{m R / I}$, where $F=\left[f_{1}, f_{2}, \ldots, f_{N}\right]$, is then generated. Each coefficient within a given $C_{m R / I}$ is then assigned to its respective matrix $f \in F$ to create the desired frame. Each resulting $f$ is then mean adjusted to create a corresponding $f^{\prime}$ using (5):

$$
f^{\prime}=f-\bar{f}
$$

where $\bar{f}$ is the mean value of the coefficients in $f$. After meanadjustment, the coefficients in each resulting image $f \in F$ are then converted to a column-wise vector and concatenated to form a two dimensional matrix $H$. PCA is then performed 
using Singular Value Decomposition (SVD) on the resulting matrix $H$ using (6):

$$
H=J \Sigma K^{T}
$$

where $J$ and $K$ are unitary matrices and the columns of $K$ are the orthonormal eigenvectors of the covariance matrix of $H . \Sigma$ is a diagonal matrix containing the eigenvalues. The eigenvectors form a basis for an eigenspace for each input frame $F$. The resulting principal components in $K$ can then be expressed in the form $K L_{m} R$ or $K L_{m} I$, where $K L_{m} R$ or $K L_{m} I$ represent the calculated eigenvectors from the mth level Curvelet transform real or imaginary frames' coefficients, respectively.

To perform matching, the total distance $D_{T}$ between a query image $q$ and every image $s \in S$, where $S$ is the dataset to be searched for comparison, is calculated. The proposed Intensity Separation Curvelet based PCA (ISC-PCA) iris recognition method is applied to both $q$ and $s$, generating eigenvectors for their respective image frames. Let $K_{q} L_{m R / I}$ and $K_{s} L_{m R / I}$ represent the resulting eigenvectors from the mth level Curvelet transform real or imaginary frames' coefficients for images $q$ and $s$, respectively. The Euclidean distance $D L_{m R / I}$ between the two sets of eigenvectors of these two frames is then calculated using (7):

$$
D L_{m R / I}=\sqrt{\sum_{i=1}^{N-1}\left(K_{q_{i}} L_{m R / I}-K_{s_{i}} L_{m R / I}\right)^{2}}
$$

The resulting Euclidean distances between the eigenvectors of all frames generated from the two images are then summed to calculate the total distance $D_{T}$. The resulting total distances are then used to find the closest image within the dataset for the input query image $q$.

\section{EXPERIMENTAL RESULTS}

The proposed Intensity Separation Curvelet based PCA (ISC-PCA) iris recognition technique was implemented using MATLAB and executed using a Windows 10 personal computer equipped with a 7th generation Intel core i7 processor, an Nvidia GTX 1080 graphics card, and a 512 GB Toshiba NVMe solid-state drive (no other applications, updates or background programs were running during the computation). The performance of the proposed algorithm was compared to the state of the art methods using the benchmark CASIA-IrisInterval, version 1.0 dataset [16]. Each iris image within this dataset is 8-bit grayscale JPEG format and has a resolution of $320 \times 280$ pixels. These images have been captured using a close-up iris camera which uses a circular Near Infrared (NIR) LED pattern for illumination. In total, the dataset contains 2639 images of 249 subjects. For this research, 100 subjects were randomly selected to serve as a testing set, with three images per subject used. The experiments began by selecting the first image of each subject to serve as a query set and the rest of the images to be a dataset. Given a particular query image, if it is correctly matched with an image in the dataset, it
TABLE I

RANK-1 ACCURACY For Single IMAge PCA AND the EIgENFACES TECHNIQUES

\begin{tabular}{|l|c|}
\hline Technique & Rank-1 Accuracy (\%) \\
\hline Single Image PCA & 39.00 \\
\hline Eigenfaces & 62.22 \\
\hline
\end{tabular}

is marked as a Rank-1 image. Finally, this process is repeated for the second and third images for each individual, with the Rank-1 accuracies averaged across all trials.

To compare the performance of the proposed technique with the state of the art PCA based and learning based techniques, experimental results were generated as follows. For the single image PCA method, PCA was applied to each input image, extracting its eigenvectors. The resulting eigenvectors for each image were then used as features to find the nearest matches. To create results for the eigenfaces PCA method, $10 \%$ of each dataset was withheld as a training set. The resulting training set was then used to calculate the eigenvectors for the 'eigenfaces', and the remaining images were projected along those eigenvectors to create eigenirises. These eigenirises were finally used to find the nearest matches for the input images using their Euclidean distances. The results for the application of single image PCA and the eigenfaces method on the benchmark image dataset are presented in Table I. From Table I, it can be seen that the performance of the eigenfaces technique exceeds that of the single image PCA method by $23.22 \%$ in Rank-1 accuracy.

The proposed ISC-PCA method was applied to the images of the CASIA-Iris-Interval dataset using two to twenty bands of constant size as detailed in Section II. It was empirically determined that the proposed technique achieves its highest performance using three level Curvelet transform with eight angles of incidence. Therefore, these parameters were used to generate experimental results presented in this paper. The percentage of correct matches was calculated for each set of bands, a subset of which are tabulated in Table II. From this table, it can be seen that the proposed ISC-PCA method significantly outperforms both the single image PCA and eigenfaces techniques. The proposed technique achieves its highest performance (95.93\% Rank-1 accuracy) using only two bins and generates $56.93 \%$ and $33.71 \%$ higher Rank1 accuracy than the single image PCA and the eigenfaces techniques, respectively. Although the Rank-1 accuracy seemingly oscillates as the number of bins increases, it ultimately stabilizes at approximately $86 \%$ from 16 bins onward. This can be explained by the fact that the distribution of the iris Curvelet subbands' coefficients is almost bimodal, therefore splitting these coefficients to more than two bins results in an uneven separation of coefficients amongst the bins and consequently causes the resulting eigenvectors to represent the data in a non-uniform manner.

To enable the reader to assess the accuracy of the ordered list of possible matches generated by the proposed ISCPCA technique, a Cumulative Matching Characteristics (CMC) 
TABLE II

RANK-1 ACCURACY FOR THE PROPOSED ISC-PCA Method

\begin{tabular}{|c|c|}
\hline Number of Bins & Rank-1 Accuracy (\%) \\
\hline $\mathbf{2}$ & $\mathbf{9 5 . 9 3}$ \\
\hline 3 & 82.96 \\
\hline 4 & 94.07 \\
\hline 5 & 90.74 \\
\hline 6 & 89.63 \\
\hline 7 & 91.85 \\
\hline 8 & 90.37 \\
\hline 9 & 82.22 \\
\hline 10 & 90.37 \\
\hline 11 & 88.52 \\
\hline 12 & 92.22 \\
\hline 13 & 91.85 \\
\hline 14 & 90.37 \\
\hline 15 & 90.00 \\
\hline 16 & 85.56 \\
\hline 17 & 86.30 \\
\hline 18 & 86.67 \\
\hline 19 & 85.93 \\
\hline 20 & 87.04 \\
\hline & \\
\hline
\end{tabular}

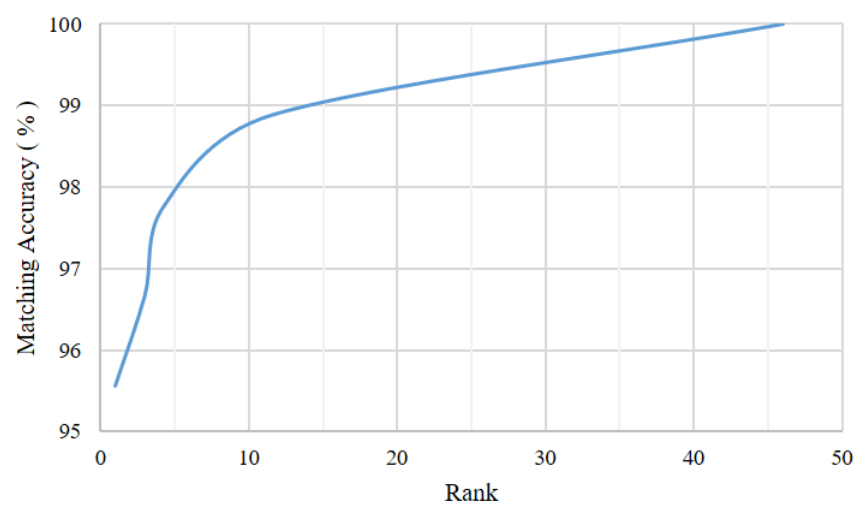

Fig. 2. Cumulative Matching Characteristics (CMC) Curve for the proposed Intensity Separation Curvelet based PCA (ISC-PCA) technique using 2 bins.

curve using two bins on the CASIA-Iris-Interval dataset is presented in Fig. 2. From this figure, it can be seen that the proposed ISC-PCA method achieves $100 \%$ matching accuracy within the top 50 ranks. Furthermore, it achieves above $99 \%$ Rank-1 accuracy within the top 15 ranks.

It should be considered that iris recognition techniques can generally be classified into two main categories: statistical based and learning based techniques. The proposed ISC-PCA algorithm falls within the statistical category, which requires significantly less computational power than learning based techniques, while it significantly outperforms other statistical based techniques such as single image PCA and eigenfaces. Furthermore, learning-based techniques utilize statistical methods such as PCA to extract features which are fed to learning based classifiers such as neural networks and support vector
TABLE III

Rank-1 Results for the Proposed ISC-PCA and State of the ART METHODS

\begin{tabular}{|l|c|}
\hline \multicolumn{1}{|c|}{ Technique } & Rank-1 Accuracy (\%) \\
\hline \multicolumn{1}{|c|}{ Statistical based Techniques } \\
\hline Single Image PCA & 39.00 \\
\hline Eigenfaces & 62.22 \\
\hline Proposed Technique & $\mathbf{9 5 . 9 3}$ \\
\hline \multicolumn{2}{|c|}{ Learning based Techniques } \\
\hline PCA and Neural Network [13] & 92.65 \\
\hline Modified Convolutional Neural Network [14] & 96.67 \\
\hline
\end{tabular}

machines. Hence the performance of learning-based methods can be improved using superior feature extraction techniques. In this paper, experimental results show that the proposed ISCPCA algorithm outperforms other techniques in feature extraction. Therefore, it has significant potential to further improve the performance of learning-based iris recognition algorithms when used as their primary feature extractor. To enable the reader to contrast the performance of the proposed ISC-PCA method against both statistical and learning based techniques, results for ISC-PCA, single image PCA, eigenfaces, PCA with Neural Network [13], and Modified Convolutional Neural Network [14] have been tabulated in III. Results with the methods presented in [3] and [4] are not presented, as those technique use a number of datasets for evaluation. The PCA with Neural Network technique applies PCA to generate eigenirises that are then fed to a neural network. This technique was tested using seven images each of 108 irises from the CASIA-Iris-Interval, version 1.0 dataset. Four images from each iris were used for training and the remaining three were used for testing. The Modified Convolutional Neural Network technique uses five samples per person from the CASIA-Iris-Interval dataset (each person has 2 images for training set, 1 images for validation set and 2 images for testing set). It uses a convolutional neural network for feature extraction, followed by fully connected layers as the learned classifier. From Table III, it can be seen that the proposed ISC-PCA technique significantly outperforms all statistical PCA based methods and the PCA and neural network based technique [13]. Furthermore, the ISCPCA algorithm gives competitive performance to that of the Modified Convolutional Neural Network algorithm [14]. The proposed ISC-PCA method achieves this competitive result at significantly less computational cost than the learning based algorithms.

\section{CONCLUSION}

This paper presented an Intensity Separation Curvelet based PCA (ISC-PCA) iris recognition technique. The proposed technique first segments the input eye image to extract the iris and converts the resulting iris into a rectangular picture. The iris image is then histogram equalized to increase its contrast and is then subjected to a Fast Discrete Curvelet Transform to split it into its subbands. The real and imaginary coefficients in the resulting subbands within each level are 
then concatenated, creating a two matrices for the coefficients in each level. The coefficients within each resulting matrix are the offset and normalized to have a range between zero and one. Equal size boundary segmentation is then applied on each resulting normalized matrix, generating a frame consisting of multiple bands. Standard Principal Component Analysis (PCA) is then applied to the resulting frame, to calculate its eigenvectors. The Euclidean distance is then calculated between the resulting eigenvectors and the eigenvectors of the images in the dataset to find the best match. Experimental results on the images of the CASIA-Iris-Interval image dataset shows that the proposed ISC-PCA technique significantly outperforms the state of the art PCA based methods. Furthermore, the results are competitive to those of the learning based techniques, yet at a much smaller computational cost. It is well known that the performance of learning based iris recognition techniques are dependent on their feature extraction methods. Consequently, the application of ISC-PCA in conjunction with learning based classification algorithms could significantly increase their performance. Finally, the proposed ISC-PCA technique could be implemented directly in hardware using existing PCA implementation algorithms such as those found using FPGA.

\section{REFERENCES}

[1] A. Ahamed and M. I. H. Bhuiyan, "Low complexity iris recognition using curvelet transform," in 2012 International Conference on Informatics, Electronics Vision (ICIEV), May 2012, pp. 548-553.

[2] L. Flom and A. Safir, "Iris recognition system," US Patent US4641349A, Feb., 1987. [Online]. Available: https://patents.google.com/patent/US4641349A/en

[3] V. Nazmdeh, S. Mortazavi, D. Tajeddin, H. Nazmdeh, and M. M. Asem, "Iris Recognition; From Classic to Modern Approaches," in 2019 IEEE 9th Annual Computing and Communication Workshop and Conference (CCWC), Jan. 2019, pp. 0981-0988.

[4] T. W. Ng, T. L. Tay, and S. W. Khor, "Iris recognition using rapid Haar wavelet decomposition," in 2010 2nd International Conference on Signal Processing Systems, vol. 1, Jul. 2010, pp. V1-820-V1-823.

[5] H. Guesmi, H. Trichili, A. M. Alimi, and B. Solaiman, "Iris verification system based on curvelet transform," in 2012 IEEE 11th International Conference on Cognitive Informatics and Cognitive Computing, Aug. 2012, pp. 226-229.

[6] R. Vyas, T. Kanumuri, and G. Sheoran, "Iris recognition using 2-D Gabor filter and XOR-SUM code," in 2016 1st India International Conference on Information Processing (IICIP), Aug. 2016, pp. 1-5.

[7] C. Chou, S. Shih, W. Chen, V. W. Cheng, and D. Chen, "Non-Orthogonal View Iris Recognition System," IEEE Transactions on Circuits and Systems for Video Technology, vol. 20, no. 3, pp. 417-430, Mar. 2010.

[8] K. Hajari, U. Gawande, and Y. Golhar, "Neural Network Approach to Iris Recognition in Noisy Environment," Procedia Computer Science, vol. 78, pp. 675-682, Jan. 2016. [Online]. Available: http://www.sciencedirect.com/science/article/pii/S1877050916001186

[9] S. S. Salve and S. P. Narote, "Iris recognition using SVM and ANN," in 2016 International Conference on Wireless Communications, Signal Processing and Networking (WiSPNET), Mar. 2016, pp. 474-478.

[10] J. Zabalza, J. Ren, M. Yang, Y. Zhang, J. Wang, S. Marshall, and J. Han, "Novel Folded-PCA for improved feature extraction and data reduction with hyperspectral imaging and SAR in remote sensing," ISPRS Journal of Photogrammetry and Remote Sensing, vol. 93, pp. 112-122, Jul. 2014. [Online]. Available: http://www.sciencedirect.com/science/article/pii/S0924271614000938

[11] E. Candes, L. Demanet, D. Donoho, and L. Ying, "Fast discrete curvelet transforms," Multiscale Modeling \& Simulation, vol. 5, no. 3, pp. 861899, 2006
[12] M. A. Turk and A. P. Pentland, "Face recognition using eigenfaces," in 1991 IEEE Computer Society Conference on Computer Vision and Pattern Recognition Proceedings, Jun. 1991, pp. 586-591.

[13] M. Abdul Hameed and E. Mattar, "Iris Recognition and Verification System Via (PCA) A Principal Component Analysis Approach," 2008.

[14] T. Le-Tien, H. Phan-Xuan, P. Nguyen-Duy, and L. Le-Ba, "Iris-based Biometric Recognition using Modified Convolutional Neural Network," in 2018 International Conference on Advanced Technologies for Communications (ATC), Oct. 2018, pp. 184-188.

[15] R. P. Wildes, "Iris recognition: an emerging biometric technology," Proceedings of the IEEE, vol. 85, no. 9, pp. 1348-1363, Sep. 1997.

[16] "CASIA Iris Image Database." [Online]. Available: http://biometrics.idealtest.org/ 